\title{
Lower bounds for blow-up time of a nonlinear viscoelastic wave equation
}

\author{
Yang Lu', Liang Fei ${ }^{2^{*}}$ and Guo Zhenhua ${ }^{1}$
}

"Correspondence: fliangmath@126.com

2Department of Mathematics, Xi An University of Science and

Technology, Xi An, 710054, China Full list of author information is available at the end of the article

\begin{abstract}
This paper deals with the blow-up for a class of nonlinear viscoelastic wave equation. Under certain conditions on the data, we construct a lower bound for the blow-up time when blow-up occurs.
\end{abstract}

MSC: $60 \mathrm{H} 15 ; 35 \mathrm{~L} 05 ; 35 \mathrm{~L} 70$

Keywords: lower bounds; blow-up; viscoelastic wave equations

\section{Introduction}

In this paper, we study the blow-up solution for the following nonlinear viscoelastic wave equation:

$$
\begin{cases}u_{t t}-\Delta u+\int_{0}^{t} g(t-s) \Delta u(s) d s+\left|u_{t}\right|^{q-2} u_{t}=|u|^{p-2} u, & (x, t) \in \Omega \times(0, T), \\ u(x, t)=0, & (x, t) \in \partial \Omega \times(0, T), \\ u(x, 0)=u_{0}(x), \quad u_{t}(x, 0)=u_{1}(x), & x \in \Omega,\end{cases}
$$

where $\Omega$ is a bounded domain in $\mathbb{R}^{n}$ with a smooth boundary $\partial \Omega, g$ is a positive function satisfying some conditions to be specified later, and

$$
2<p, q \leq \begin{cases}\infty, & \text { if } n=1,2 \\ \frac{2 n-2}{n-2}, & \text { if } n \geq 3\end{cases}
$$

The blow-up properties of the solution to (1.1) has been studied by many authors (see [15]). For instance, Messaoudi [2] studied (1.1) and proved a blow-up result for solutions with negative initial energy if $p>q \geq 2$ and a global result for $2 \leq p \leq q$. This result has been later improved by the same author in [3] to accommodate certain solutions with positive initial energy. In [4], Song and Zhong considered (1.1) for strong damping $-\Delta u_{t}$ and proved a blow-up result for solutions with positive initial energy by using the ideas of the 'potential well' theory introduced by Payne and Sattinger [6]. Wang [5] has investigated a sufficient conditions of the initial data with arbitrarily positive initial energy such that the corresponding solution of (1.1) with $q=2$ blows up in finite time. For related results, we refer the reader to [7-10].

When blow-up occurs, the blow-up time $T^{*}$ cannot usually be computed exactly. It is therefore of great importance in practice to determine lower and upper bounds for $T^{*}$.

(c) 2015 Lu et al. This article is distributed under the terms of the Creative Commons Attribution 4.0 International License (http://creativecommons.org/licenses/by/4.0/), which permits unrestricted use, distribution, and reproduction in any medium, provided you give appropriate credit to the original author(s) and the source, provide a link to the Creative Commons license, and indicate if changes were made. 
The aim of this note is to derive a lower bound for $T^{*}$ when blow-up occurs. We point out that it is, in general, very hard to obtain a lower bound estimate for viscoelastic wave equation problems, for the method to estimate the derivative of the control functional in parabolic cases is no longer effective and the memory part makes it difficult to estimate the energy. Our method is based on a first-order differential inequality technique for a suitably defined auxiliary function and makes use of some Sobolev-type inequality.

Before stating our main result, let us recall some results on the local existence, uniqueness, and blow-up of the solution

Theorem 1.1 (see [3]) Let $\left(u_{0}(x), u_{1}(x)\right) \in H_{0}^{1}(\Omega) \times L^{2}(\Omega)$ and $p$, q satisfy condition (1.2). Let $g \in C^{1}[0, \infty)$ be a non-negative and non-increasing function satisfying

$$
1-\int_{0}^{\infty} g(s) d s=l>0 .
$$

Then problem (1.1) has a unique local solution

$$
u \in C\left(\left[0, T_{m}\right) ; H_{0}^{1}(\Omega)\right), \quad u_{t} \in C\left(\left[0, T_{m}\right) ; L^{2}(\Omega)\right) \cap L^{q}\left(\Omega \times\left(0, T_{m}\right)\right)
$$

for some $T_{m}>0$.

Remark 1.1 Condition (1.3) is necessary to guarantee the hyperbolicity and wellposedness of system (1.1).

Let $\lambda$ be the best constant of the Sobolev embedding $H_{0}^{1}(\Omega) \hookrightarrow L^{p}(\Omega)$ and $\beta=\lambda / l^{\frac{1}{2}}$. We set

$$
\alpha=\beta^{-\frac{p}{p-2}}, \quad E_{1}=\left(\frac{1}{2}-\frac{1}{p}\right) \alpha^{2} .
$$

Define the energy functional $E(t)$ associated to our system (1.1),

$$
E(t)=\frac{1}{2}\left\|u_{t}(t)\right\|_{2}^{2}+\frac{1}{2}\left(1-\int_{0}^{t} g(s) d s\right)\|\nabla u(t)\|_{2}^{2}+\frac{1}{2}(g \circ \nabla u)(t)-\frac{1}{p}\|u\|_{p}^{p},
$$

where

$$
g \circ w(t)=\int_{0}^{t} g(t-s)\|w(s)-w(t)\|_{2}^{2} d s .
$$

Moreover, we assume that $g$ satisfies

$$
\int_{0}^{\infty} g(s) d s<\frac{p^{2}-2 p}{p^{2}-2 p+1}
$$

Then we have the following blow-up result.

Theorem 1.2 (see [3]) Assume that p, $q$ satisfy condition (1.2) and $g$ satisfies (1.3) and (1.4). If $p>q$ and the initial data $\left(u_{0}, u_{1}\right)$ satisfies

$$
E(0)>E_{1}, \quad\left\|\nabla u_{0}\right\|_{2}>\alpha,
$$

then any solution of (1.1) blows up in finite time. 


\section{The main result}

In this section, we switch to discuss the lower bound of the blow-up time for the blow-up solution of (1.1). Before we state and prove our main result, we need the following lemma.

Lemma 2.1 Suppose that (1.2), (1.3), and (1.4) hold. Let u be a solution of (1.1). Then energy functional $E(t)$ is non-increasing, that is, $E^{\prime}(t) \leq 0$.

Proof By multiplying (1.1) by $u_{t}$ and integrating over $\Omega$, we obtain

$$
\begin{aligned}
& \frac{d}{d t}\left(\frac{1}{2}\left\|u_{t}\right\|_{2}^{2}+\frac{1}{2}\|\nabla u\|_{2}^{2}-\frac{1}{p}\|u\|_{p}^{p}\right)-\int_{0}^{t} g(t-s) \int_{\Omega} \nabla u(s) \cdot \nabla u_{t}(t) d x d s \\
& \quad=-\int_{0}^{t}\left\|u_{t}(s)\right\|_{q}^{q}
\end{aligned}
$$

for any regular solution. This result remains valid for weak solutions by a simple density argument. For the last term on the left side of (2.1), we have

$$
\begin{aligned}
& \int_{0}^{t} g(t-s) \int_{\Omega} \nabla u_{t}(t) \cdot \nabla u(s) d x d s \\
& \quad=\int_{0}^{t} g(t-s) \int_{\Omega} \nabla u_{t}(t)(\nabla u(s)-\nabla u(t)) d x d s+\int_{0}^{t} g(t-s) \int_{\Omega} \nabla u_{t}(t) \cdot \nabla u(t) d x d s \\
& \quad=-\frac{1}{2} \int_{0}^{t} g(t-s) \frac{d}{d t} \int_{\Omega}|\nabla u(s)-\nabla u(t)|^{2} d x d s+\frac{1}{2} \int_{0}^{t} g(s) \frac{d}{d t} \int_{\Omega}|\nabla u(t)|^{2} d x d s \\
& \quad=\frac{1}{2} \frac{d}{d t}\left(\int_{0}^{t} g(s) d s\|\nabla u(t)\|_{2}^{2}-(g \circ \nabla u)(t)\right)+\frac{1}{2}\left(g^{\prime} \circ \nabla u\right)(t)-\frac{1}{2} g(t)\|\nabla u(t)\|_{2}^{2} .
\end{aligned}
$$

Inserting (2.2) into (2.1), we get

$$
E^{\prime}(t)=-\int_{0}^{t}\left\|u_{t}(s)\right\|_{q}^{q}+\frac{1}{2}\left(g^{\prime} \circ \nabla u\right)(t)-\frac{1}{2} g(t)\|\nabla u(t)\|_{2}^{2} \leq 0
$$

where we also use $g$ being non-negative and non-increasing function.

Theorem 2.2 Assume that the conditions in Theorem 1.2 hold. Let $u(x, t)$ be the solution of problem (1.1), which blows up at a finite time $T^{*}$. Then

$$
T^{*} \geq \int_{F(0)}^{\infty} \frac{1}{C_{3} y^{k}+y+C_{4}} d y,
$$

where the constants $C_{3}, C_{4}$, and the exponent $k$ will be defined in (2.9), and $F(0)=$ $\int_{\Omega}\left|u_{0}\right|^{p} d x$.

Proof Define $F(t)=\int_{\Omega}|u(t)|^{p} d x$. Then

$$
F^{\prime}(t)=p \int_{\Omega}|u|^{p-2} u u_{t} d x \leq \frac{p}{2}\left(\int_{\Omega}|u|^{2 p-2} d x+\int_{\Omega}\left|u_{t}\right|^{2} d x\right) .
$$

To estimate the first term on the right side of inequality (2.3), we consider the following two cases. 
Case $1.2<p \leq \frac{2 n}{n-1}$. Let $\gamma=2 p-2, \mu=n(p-2), 2^{*}=\frac{2 n}{n-2}$. Applying Hölder's inequality and the embedding inequality, we have

$$
\int_{\Omega}|u|^{\gamma} d x=\int_{\Omega}|u|^{\gamma \theta}|u|^{\gamma(1-\theta)} d x \leq\left(\int_{\Omega}|u|^{\mu} d x\right)^{\frac{\gamma \theta}{\mu}}\left(\int_{\Omega}|u|^{2^{*}} d x\right)^{\frac{\gamma(1-\theta)}{2^{*}}}
$$

where $\theta$ satisfies

$$
\frac{\gamma \theta}{\mu}+\frac{\gamma(1-\theta)}{2^{*}}=1
$$

A straightforward computation shows

$$
\begin{aligned}
& \theta=\frac{1-\frac{\gamma}{2^{*}}}{\frac{\gamma}{\mu}-\frac{\gamma}{2^{*}}}=\frac{\mu\left(2^{*}-\gamma\right)}{\gamma\left(2^{*}-\mu\right)}, \\
& \frac{\gamma \theta}{\mu}=\frac{2^{*}-\gamma}{2^{*}-\mu}=\frac{2}{n}, \quad \frac{\gamma-\theta \gamma}{2^{*}}=1-\frac{2}{n},
\end{aligned}
$$

and then we have

$$
\begin{aligned}
\|u\|_{\gamma}^{\gamma} & \leq\|u\|_{\mu}^{\gamma \theta}\|u\|_{2^{*}}^{\gamma(1-\theta)}=\|u\|_{\mu}^{\frac{2 \mu}{n}}\|u\|_{2^{*}}^{2} \\
& \leq C_{*}^{2}\left(1+|\Omega|^{\frac{2(p-\mu)}{n p}}\right)\|u\|_{p}^{\frac{2 \mu}{n}}\|\nabla u\|_{2}^{2} \\
& \leq C_{*}^{2}\left(1+|\Omega|^{\frac{2(p-\mu)}{n p}}\right)\left(\|u\|_{p}^{\frac{2 \mu}{n} \cdot s}+\|\nabla u\|_{2}^{2 t}\right) \\
& \leq C_{1}\left(\|u\|_{p}^{p}+\|\nabla u\|_{2}^{2}\right)^{k_{1}},
\end{aligned}
$$

where we have used the Hölder inequality,

$$
\|u\|_{\mu}^{\frac{2 \mu}{n}} \leq|\Omega|^{\frac{2(p-\mu)}{n p}}\|u\|_{p}^{\frac{2 \mu}{n}} \leq\left(1+|\Omega|^{\frac{2(p-\mu)}{n p}}\right)\|u\|_{p}^{\frac{2 \mu}{n}}
$$

and

$$
\|u\|_{2^{*}} \leq C_{*}\|\nabla u\|_{2}
$$

here $C_{*}$ is the best constant of the Sobolev embedding $H_{0}^{1}(\Omega) \hookrightarrow L^{2^{*}}(\Omega) ; \frac{1}{s}+\frac{1}{t}=1$, letting $t=\frac{2 \mu}{p n} s$, from which we can deduce $k_{1}=\frac{3 p-4}{p}, C_{1}=C_{*}^{2}\left(1+|\Omega|^{\frac{2(p-\mu)}{n p}}\right)$.

Case 2. $\frac{2 n}{n-1}<p \leq \frac{2(n-1)}{n-2}$. Following the lines of the proof of inequality (2.4), we have

$$
\begin{aligned}
\|u\|_{\gamma}^{\gamma} \leq|\Omega|^{1-\frac{\gamma}{2^{*}}}\|u\|_{2^{*}}^{\gamma} & \leq C_{*}^{\gamma}\left(1+|\Omega|^{1-\frac{\gamma}{2^{*}}}\right)\|\nabla u\|_{2}^{\gamma} \\
& \leq C_{*}^{\gamma}\left(1+|\Omega|^{1-\frac{\gamma}{2^{*}}}\right)\left(\|\nabla u\|_{2}^{\gamma}+\|u\|_{p}^{p(p-1)}\right) \\
& \leq C_{2}\left(\|u\|_{p}^{p}+\|\nabla u\|_{2}^{2}\right)^{k_{2}},
\end{aligned}
$$

with $k_{2}=p-1, C_{2}=C_{*}^{\gamma}\left(1+|\Omega|^{1-\frac{\gamma}{2^{*}}}\right)$.

From Lemma 2.1, we have

$$
E(t) \leq E(0)=\frac{1}{2}\left\|u_{1}\right\|_{2}^{2}+\frac{1}{2}\left\|\nabla u_{0}\right\|_{2}^{2}-\frac{1}{p}\left\|u_{0}\right\|_{p}^{p}=E_{0}, \quad t \in\left[0, T^{*}\right) .
$$


Recalling the definition of $E(t),(1.4)$, and (2.6), we have

$$
\begin{aligned}
& \left\|u_{t}(t)\right\|_{2}^{2}+\frac{1}{(p-1)^{2}}\|\nabla u(t)\|_{2}^{2}+g \circ u(t) \\
& \leq\left\|u_{t}(t)\right\|_{2}^{2}+\left(1-\int_{0}^{t} g(s) d s\right)\|\nabla u(t)\|_{2}^{2}+g \circ u(t) \\
& =\frac{2}{p}\|u(t)\|_{p}^{p}+2 E(t) \leq \frac{2}{p} F(t)+2 E_{0} .
\end{aligned}
$$

Combining (2.3)-(2.7), we get

$$
\begin{aligned}
F^{\prime}(t) & \leq \frac{p}{2}\left(C_{i}\left(\|u\|_{p}^{p}+\|\nabla u\|_{2}^{2}\right)^{k_{i}}+\frac{2}{p} F(t)+2 E_{0}\right) \\
& \leq \frac{p}{2}\left(C_{i}\left(F(t)+C_{0}\left(\frac{2}{p} F(t)+2 E_{0}\right)\right)^{k_{i}}+\frac{2}{p} F(t)+2 E_{0}\right) \\
& \leq \frac{p}{2}\left(C_{i}\left(\left(1+\frac{2 C_{0}}{p}\right) F(t)+2 C_{0} E_{0}\right)^{k_{i}}+\frac{2}{p} F(t)+2 E_{0}\right) \\
& \leq \frac{p C_{i}}{2} 2^{k_{i}-1}\left(\left(1+\frac{2 C_{0}}{p}\right)^{k_{i}} F(t)^{k_{i}}+\left(2 C_{0} E_{0}\right)^{k_{i}}\right)+F(t)+p E_{0} \\
& =C_{3} F(t)^{k_{i}}+F(t)+C_{4},
\end{aligned}
$$

where

$$
\begin{aligned}
& C_{0}=\frac{1}{1-\int_{0}^{\infty} g(s) d s} \\
& C_{3}=\frac{p C_{i} 2^{k_{i}}}{4}\left(1+\frac{2 C_{0}}{p}\right)^{k_{i}}, \\
& C_{4}=p E_{0}+\frac{p C_{i}}{4}\left(4 C_{0} E_{0}\right)^{k_{i}}, \quad i=1,2 .
\end{aligned}
$$

Applying Theorem 1.2, we have

$$
\lim _{t \rightarrow T^{*}} \int_{\Omega}|u|^{p} d x=+\infty
$$

According to (2.8) and (2.10), we obtain

$$
\int_{F(0)}^{\infty} \frac{1}{C_{3} y^{k}+y+C_{4}} d y \leq T^{*}
$$

\section{Competing interests}

The authors declare that they have no competing interests.

Authors' contributions

The article is a joint work of the three authors, who contributed equally to the final version of the paper. All authors read and approved the final manuscript.

\section{Author details}

${ }^{1}$ Department of Mathematics, Northwest University, Xi An, 710069, China. ${ }^{2}$ Department of Mathematics, Xi An University of Science and Technology, Xi An, 710054, China. 


\section{Acknowledgements}

The authors are indebted to the referee for giving some important suggestions which improved the presentations of this paper. This work is supported in part by China NSF Grant No. 11501442, the China Postdoctoral Science Foundation Grant No. 2013M540767, the Shanxi Provincial Postdoctoral Science Foundation, the scientific research program funded by Shanxi Provincial education department No. 14JK1474, and the doctor scientific research start fund project of Xi An University of Science and Technology Grant No. 2014QDJ042.

Received: 17 July 2015 Accepted: 10 November 2015 Published online: 01 December 2015

\section{References}

1. Kafini, M, Messaoudi, SA: A blow-up result in a Cauchy viscoelastic problem. Appl. Math. Lett. 21, 549-553 (2008)

2. Messaoudi, SA: Blow up and global existence in a nonlinear viscoelastic wave equation. Math. Nachr. 260, 58-66 (2003)

3. Messaoudi, SA: Blow up of positive-initial-energy solutions of a nonlinear viscoelastic hyperbolic equation. J. Math. Anal. Appl. 320, 902-915 (2006)

4. Song, HT, Zhong, CK: Blow-up of solutions of a nonlinear viscoelastic wave equation. Nonlinear Anal. 11, 3877-3883 (2010)

5. Wang, YJ: A global nonexistence theorem for viscoelastic equations with arbitrarily positive initial energy. Appl. Math. Lett. 22, 1394-1400 (2009)

6. Payne, L, Sattinger, D: Saddle points and instability on nonlinear hyperbolic equations. Isr. J. Math. 22, 273-303 (1975)

7. Levine, HA, Ro Park, S: Global existence and global nonexistence of solutions of the Cauchy problem for a nonlinearly damped wave equation. J. Math. Anal. Appl. 228, 181-205 (1998)

8. Vitillaro, E: Global nonexistence theorems for a class of evolution equations with dissipation. Arch. Ration. Mech. Anal. 149, 155-182 (1999)

9. Yang, ZJ: Existence and asymptotic behavior of solutions for a class of quasi-linear evolution equations with non-linear damping and source terms. Math. Methods Appl. Sci. 25, 795-814 (2002)

10. Messaoudi, SA, Said-Houari, B: Blow up of solutions of a class of wave equations with nonlinear damping and source terms. Math. Methods Appl. Sci. 27, 1687-1696 (2004)

\section{Submit your manuscript to a SpringerOpen ${ }^{\ominus}$ journal and benefit from:}

- Convenient online submission

- Rigorous peer review

- Immediate publication on acceptance

- Open access: articles freely available online

- High visibility within the field

- Retaining the copyright to your article 